\title{
Optimized determination of the polarized Bjorken sum rule in pQCD
}

\author{
D. Kotlorz $\oplus^{1,2, *}$ and S. V. Mikhailov ${ }^{2, \dagger}$ \\ ${ }^{1}$ Department of Physics, Opole University of Technology, 45-758 Opole, Proszkowska 76, Poland \\ ${ }^{2}$ Bogoliubov Laboratory of Theoretical Physics, JINR, 141980 Dubna, Russia
}

(Received 17 October 2018; published 11 September 2019)

\begin{abstract}
We present the method of numerical optimization for the perturbative series using the renormalization group in quantum chromodynamics. We apply our approach to the perturbation series in $\alpha_{s}$ for the coefficient function $C_{\mathrm{Bjp}}\left(\alpha_{s}\right)$ of the Bjorken sum rule for the polarized deep inelastic lepton-hadron scattering. We optimize the Bjorken sum rule value, $\Gamma_{1}^{\mathrm{p}-\mathrm{n}}$, at the COMPASS, SLAC, and JLab kinematics and compare the obtained results with the experimental measurements and also with the truncated Bjorken sum rule predictions.
\end{abstract}

DOI: $10.1103 /$ PhysRevD.100.056007

\section{INTRODUCTION}

One of the foundations of quantum chromodynamics (QCD) is the renormalization group (RG) equation. The latter defines the running of the coupling $\alpha_{s}\left(Q^{2}\right)$ that characterizes the strong interaction of quarks and gluons. Understanding the behavior of $\alpha_{s}$ with the scale of the virtual momenta $Q^{2}$ allows us to describe hadronic interactions at both short and long distances. The long-distance domain at low $Q^{2}$ is characteristic of quark confinement and processes of hadronization, and the short-distance domain, at high $Q^{2}$, involves perturbative methods of QCD (pQCD). The calculation of the 1-loop $\beta$-function in pQCD over 40 years ago enabled the discovery of asymptotic freedom. Since then, tremendous progress has been made in perturbative calculations in QCD. Particularly, the Bjorken sum rule (BSR) for the polarized deep inelastic lepton-hadron scattering (DIS) [1,2], providing fundamental spin predictions of the nucleon, has been studied in detail, both theoretically and experimentally. The radiative corrections to BSR in the strong coupling constant $\alpha_{s}$ of order $O\left(\alpha_{s}^{n}\right), n=1, \ldots, 4$ were obtained in [3-6], respectively. In order to optimize the perturbative series in $\alpha_{s}$ of a physical observable, various methods can be used. One of them is the Brodsky-LepageMackenzie (BLM) approach [7], later on developed in two different approaches: the sequential BLM (seBLM),

\footnotetext{
*dorota@theor.jinr.ru

†mikhs@theor.jinr.ru
}

Published by the American Physical Society under the terms of the Creative Commons Attribution 4.0 International license. Further distribution of this work must maintain attribution to the author(s) and the published article's title, journal citation, and DOI. Funded by SCOAP. e.g., $[8,9]$, and the principle of maximal conformality (PMC), e.g., [10] and references therein.

In this paper, we present another method of optimization applied to the coefficient function $C_{\mathrm{Bjp}}\left(\alpha_{s}\right)$ for BSR predictions. In the next section, we discuss and fix the criteria for the optimized analysis of the $C_{\mathrm{Bjp}}\left(\alpha_{s}\right)$ within the perturbation QCD and RG. Following these criteria, in Sec. III, we find numerically the admissible domains for the corresponding new normalization scales $\mu^{2}\left(\mu^{2} \neq Q^{2}\right)$. In Sec. IV, the results of optimization are presented and discussed. We optimize the perturbation expansion for the BSR at the COMPASS, SLAC, and Jefferson Lab (JLab) kinematics and compare the obtained results with the experimental measurements and also with our predictions based on the truncated Bjorken sum rule (tBSR) approach [11,12].

\section{RENORMALIZATION GROUP ANALYSIS OF QCD PT SERIES FOR BJORKEN SUM RULE}

For decades, perturbative QCD has been a powerful tool in understanding the hadron structure. In the analysis, the hadronic observables, like DIS sum rules, are expanded into the power series in the strong coupling $\alpha_{s}$, providing a robust test of the perturbative theory (PT). The Bjorken sum rule [1,2], $\Gamma_{1}^{p-n}\left(Q^{2}\right)$, which is a fundamental sum rule for polarized DIS at hard momentum transfer $q:-q^{2}=$ $Q^{2}$ and a rigorous $\mathrm{pQCD}$ prediction, is essential for describing the nucleon spin structure. In the limit $Q^{2} \rightarrow \infty$, the BSR relates the difference between the first moments of the proton, $g_{1}^{p}$, and the neutron, $g_{1}^{p}$, spin structure functions to the nucleon axial charge, $g_{A}$, $\Gamma_{1}^{p-n}=\left|g_{A}\right| / 6$. Since in real experiments $Q^{2}$ cannot reach infinity, the QCD analysis of the BSR involves both the perturbative leading-twist and the nonperturbative highertwist (HT) corrections. Thus, away from the large $Q^{2}$ limit, the $Q^{2}$ dependence of the polarized BSR is given by 
$\Gamma_{1}^{\mathrm{th}}\left(Q^{2}\right)=\Gamma_{1}^{p}\left(Q^{2}\right)-\Gamma_{1}^{n}\left(Q^{2}\right)=\left|\frac{g_{A}}{6}\right| C_{\mathrm{Bjp}}\left(a_{s}\right)+\sum_{i=2}^{\infty} \frac{\mu_{2 i}^{p-n}}{Q^{2 i-2}}$,

where $C_{\mathrm{Bjp}}\left(a_{s}\right)$ is the leading-twist nonsinglet coefficient function (c.f.) including radiative corrections obtained within the $\overline{\mathrm{MS}}$ scheme and is known to 4 loops [3-6]. The HT contribution is a series in power of $1 / Q^{2}$, where $\mu_{2 i}^{p-n}$ are the effective scales of the power corrections whose effects become essential in the small and moderate $Q^{2}$ region. Below, we will investigate the $\mathrm{QCD}$ radiative corrections to $C_{\mathrm{Bjp}}\left(a_{s}\right)$ based on the renormalization group transform.

\section{A. The problem of PT optimization for coefficient function $C_{\mathrm{B} \text { jp }}\left(a_{s}\right)$}

The perturbation expansion for the c.f. $C_{\mathrm{Bjp}}\left(a_{s}\right)$ reads

$$
\begin{aligned}
C_{\mathrm{Bjp}}\left(\frac{Q^{2}}{\mu^{2}}, a_{s}\left(\mu^{2}\right)\right)= & 1+c_{1}\left(a_{s}\left(\mu^{2}\right)+c_{2} a_{s}^{2}\left(\mu^{2}\right)\right. \\
& \left.+c_{3} a_{s}^{3}\left(\mu^{2}\right)+c_{4} a_{s}^{4}\left(\mu^{2}\right)+\ldots\right),
\end{aligned}
$$

where the coefficients $c_{i}=c_{i}\left(Q^{2} / \mu^{2}\right)$ are calculated in the $\overline{\mathrm{MS}}$ scheme and are normalized by the first coefficient $c_{1}=-3 C_{\mathrm{F}}=-4$; the running $\mathrm{QCD}$ coupling $a_{s}$ is $a_{s}\left(\mu^{2}\right)=\boldsymbol{\alpha}_{s}\left(\mu^{2}\right) /(4 \pi)$. For the default condition $\mu^{2}=Q^{2}$, the coefficients $c_{i} \equiv c_{i}(1)$ are the numbers presented in the Appendix $\mathrm{A}$ in different forms,

$$
\begin{aligned}
C\left(a_{s}\left(\mu^{2}\right)\right) \equiv & C_{\mathrm{Bjp}}\left(1, a_{s}\left(\mu^{2}\right)\right) \\
= & 1-4\left(a_{s}\left(\mu^{2}\right)+13 a_{s}^{2}\left(\mu^{2}\right)+221.6 a_{s}^{3}\left(\mu^{2}\right)\right. \\
& \left.+6553.7 a_{s}^{4}\left(\mu^{2}\right)+\ldots\right) .
\end{aligned}
$$

The numerical estimates in Eq. (2b) are taken at the number of active quarks $n_{f}=4$; see Eq. (A6). For the character reference scale of BSR measurements near the $\tau$-lepton mass, $\mu^{2}=m_{\tau}^{2} \approx 3.16 \mathrm{GeV}^{2}, a_{s}\left(m_{\tau}^{2}\right) \approx 0.332 /(4 \pi) \approx 0.0264$ [here $\alpha_{s}\left(m_{\tau}^{2}\right)=0.332 \pm 0.005$ (exp) \pm 0.015 (theor) [13] ], one obtains for the series in Eq. (2b) the estimate

$$
\begin{aligned}
C\left(a_{s}\left(m_{\tau}^{2}\right)\right) \equiv & C_{\mathrm{Bjp}}\left(1, a_{s}\left(m_{\tau}^{2}\right)\right) \\
= & 1-4(0.0264+0.0090+0.0041 \\
& +0.0032+\ldots) \\
= & 1-4(0.0428) .
\end{aligned}
$$

One can see that the radiative corrections are significant, being about $-17 \%$ of the Born term in Eq. (3), and the convergence of the series is not very good. Below, we perform an optimization of the partial sum in Eq. (2a) by choosing an appropriate new normalization scale $\mu \rightarrow \mu^{\prime}$ and following the renormalization group transform. The value of the partial sum for the series in Eq. (2) as well as the values of its separate terms start to change at the variation of the renormalization scale $\mu^{2}$ around the default scale $Q^{2}$. This is the inevitable effect of the series truncation which we will use for optimization. Our goal is to make smaller the total value of radiative corrections in Eq. (2a), keeping simultaneously some natural hierarchy of the coefficients $c_{i}$ for appropriate convergence, using for this purpose the variation of a scale $\mu$. The corresponding approach goes back to the generalization of the BLM [7] method, which was suggested in $[8,9]$ for the RG invariant quantities. The approach is based on the $\{\beta\}$-expansion for the PT coefficients [8] $]^{1}$, here they are presented for $c_{i}$ in Appendix A, which allows us to derive the intrinsic structure of $c_{i}$ in connection with charge renormalization in great detail. An alternative approach to the PT optimization, named PMC (see [15]), is elaborated and applied to BSR in [10]; we will discuss its results in Sec. IV. However, it is not necessary to know all the details of the series structure to solve a practical optimization of this series. Here, we will avoid the details of $\{\beta\}$-expansion (different for different approaches) and will not discuss them, but, instead, we propose a direct numerical method to deal with the partial sums of the series.

In other words, following the RG transform, we will reorganize four successive orders of radiative corrections in the parentheses in the rhs of Eq. (3a) to make their sum minimal. In the next subsection, we will remind the reader the required elements of the formalism for transformation of the expansion coefficients for any RG invariant (RGI) quantity.

\section{B. Parametrization of the RG transformation}

We consider the transformation of the coefficients $c_{i}$ of the RGI quantity $C_{\mathrm{Bjp}}\left(a_{s}\right)$ under the change of the normalization scale $\mu \rightarrow \mu^{\prime}$. Let $a_{s}=\bar{a}_{s}(t)$ and $a_{s}^{\prime}=\bar{a}_{s}\left(t^{\prime}\right)$ be the solutions of the RG equation for the QCD charge with logarithmic argument $t=\ln \left(\mu^{2} / \Lambda_{q c d}^{2}\right)$ at the same integration constant $\Lambda_{q c d}^{2}$. Reexpanding the running coupling $\bar{a}_{s}(t)=a_{s}\left(\Delta, a_{s}^{\prime}\right)$ in terms of $\Delta=t-t^{\prime}=\ln \left(\mu^{2} / \mu^{\prime 2}\right)$ and the coupling $a_{s}^{\prime}$, we obtain

$$
\begin{aligned}
a_{s} & =a_{s}\left(\Delta, a_{s}^{\prime}\right) \\
& =\left.\exp \left[-\Delta \beta\left(\bar{a}_{s}\right) \partial_{\bar{a}_{s}}\right] \bar{a}_{s}\right|_{\bar{a}_{s}=a_{s}^{\prime}} \\
& =a_{s}^{\prime}-\beta\left(a_{s}^{\prime}\right) \frac{\Delta}{1 !}+\beta\left(a_{s}^{\prime}\right) \partial_{a_{s}^{\prime}} \beta\left(a_{s}^{\prime}\right) \frac{\Delta^{2}}{2 !}+\ldots .
\end{aligned}
$$

This is the way to write the RG solution for $\bar{a}(t)$ through the operator $\left.\exp \left(-\Delta \beta(a) \partial_{a}\right)[\ldots]\right|_{a=a^{\prime}}$ (see [8,9] and references

\footnotetext{
${ }^{1}$ Another approach to construction of the $\{\beta\}$-expansion was suggested in [14], but their results are numerically close.
} 
TABLE I. The first few elements of the matrix $B_{i j}$. New PT coefficients $c_{i}^{\prime}=B_{i j} c_{j}$.

\begin{tabular}{lccc}
\hline \hline $\mathbf{1}$ & 0 & 0 & 0 \\
$-\beta_{0} \Delta_{0}$ & $\mathbf{1}$ & 0 & 0 \\
$-\beta_{0}^{2} \Delta_{1}-\beta_{1} \Delta_{0}+\beta_{0}^{2} \Delta_{0}^{2}$ & $-2 \beta_{0} \Delta_{0}$ & $\mathbf{1}$ & 0 \\
$-\beta_{0}^{3} \Delta_{2}-\beta_{2} \Delta_{0}-\beta_{0} \beta_{1} \Delta_{1}+\frac{3}{2} \beta_{0} \beta_{1} \Delta_{0}+2 \beta_{0}^{3} \Delta_{0} \Delta_{1}$ & $-2 \beta_{0}^{2} \Delta_{1}-2 \beta_{1} \Delta_{0}+3 \beta_{0}^{2} \Delta_{0}^{2}$ & $-3 \beta_{0} \Delta_{0}$ & $\mathbf{1}$ \\
\hline \hline
\end{tabular}

therein). The shift $\Delta$ of the logarithmic scale in Eq. (4) can be expanded in its turn in the perturbation series in powers of the rescaled charge $a_{s}^{\prime} \beta_{0}[8]$ :

$$
\begin{aligned}
t^{\prime} & \equiv t-\Delta, \\
\Delta & \equiv \Delta\left(a_{s}^{\prime}\right)=\Delta_{0}+a_{s}^{\prime} \beta_{0} \Delta_{1}+\left(a_{s}^{\prime} \beta_{0}\right)^{2} \Delta_{2}+\ldots,
\end{aligned}
$$

where the argument of the new coupling $a_{s}^{\prime}$ depends on $\Delta$, i.e., $a_{s}^{\prime} \equiv \bar{a}_{s}\left(t^{\prime}\right)=a_{s}\left(t-\Delta\left(a_{s}^{\prime}\right)\right)$. Reexpansion $a_{s}$ in terms of $a_{s}^{\prime}$ and $\Delta_{i}$ leads to rearrangement of the perturbation series for c.f. $C_{\mathrm{Bjp}}\left(a_{s}\right)=\sum_{i} a_{s}^{i} c_{i} \rightarrow \sum_{i}\left(a_{s}^{\prime}\right)^{i} c_{i}^{\prime}$. The new primed coefficients $c_{i}^{\prime}$ there can be expressed as $c_{i}^{\prime}=B_{i j} c_{j}$, where $B_{i j}$ is a triangular matrix presented in Table I. In this notation, $C_{\mathrm{Bjp}}$ from Eq. (2b) transforms to

$$
C_{\mathrm{Bjp}}\left(a_{s}\right)=\sum_{i \geq 0} a_{s}^{i} c_{i} \rightarrow \sum_{i \geq 0}\left(a_{s}^{\prime}\right)^{i} c_{i}^{\prime}=1+\sum_{i, j \geq 1}\left(a_{s}^{\prime}\right)^{i} B_{i j} c_{j}
$$

when the normalization scale $\mu$ is transformed $\mu \rightarrow \mu^{\prime}$. The elements $B_{i j}$ appear as a composition of transforms in Eq. (4) taken together with the expansion in Eq. (5).

Below, in the square brackets we write explicitly the elements of the triangle matrix $B$ :

$$
\begin{aligned}
& a_{s}^{1} \cdot c_{1} \rightarrow a_{s}^{\prime 1} \cdot\left[c_{1}^{\prime}=1\right] \\
& a_{s}^{2} \cdot c_{2} \rightarrow a_{s}^{\prime 2} \cdot\left[c_{2}^{\prime}\left(\Delta_{0}\right)=c_{2}-1 \cdot \beta_{0} \Delta_{0}\right] \\
& a_{s}^{3} \cdot c_{3} \rightarrow a_{s}^{\prime 3} \cdot\left[c_{3}^{\prime}\left(\Delta_{0}, \Delta_{1}\right)=c_{3}-c_{2} \cdot 2 \beta_{0} \Delta_{0}-1 \cdot\left(\beta_{1} \Delta_{0}-\beta_{0}^{2} \Delta_{0}^{2}+\beta_{0}^{2} \Delta_{1}\right)\right] ; \\
& a_{s}^{4} \cdot c_{4} \rightarrow a_{s}^{\prime 4} \cdot\left[c_{4}^{\prime}\left(\left\{\Delta_{i}\right\}_{0}^{2}\right)=c_{4}-c_{3} \cdot 3 \beta_{0} \Delta_{0}-c_{2} \cdot\left(2 \beta_{1} \Delta_{0}-3 \beta_{0}^{2} \Delta_{0}^{2}+2 \beta_{0}^{2} \Delta_{1}\right)-\right. \\
& \left.-1 \cdot\left(\beta_{2} \Delta_{0}+\beta_{0} \beta_{1} \Delta_{1}-\frac{3}{2} \beta_{0} \beta_{1} \Delta_{0}^{2}-2 \beta_{0}^{3} \Delta_{0} \Delta_{1}+\beta_{0}^{3} \Delta_{2}\right)\right] \\
& \text {. } \\
& a_{s}^{n} \cdot c_{n} \rightarrow a_{s}^{\prime n} \cdot\left[c_{n}^{\prime}\left(\left\{\Delta_{i}\right\}_{0}^{n-2}\right)=c_{n}-c_{n-1} \cdot(n-1) \beta_{0} \Delta_{0}-\ldots\right] .
\end{aligned}
$$

New coefficients $c_{n}^{\prime}$ in Eq. (7) depend on the fitted parameters $\Delta_{i}$. The different approaches based on different $\{\beta\}$-expansion for $c_{i}$ tell us how to deal with $\Delta_{i}$ to fix these $c_{i}^{\prime}$. At this point it is instructive to recall the standard BLM [7] procedure which deals with $O\left(a_{s}^{2}\right)$ order and is based on the decomposition $c_{2}=\beta_{0} \cdot c_{2}[1]+c_{2}[0]$. BLM fixes the scale $\Delta_{0}$ in Eq. (7a) by the requirement $\Delta_{0}=c_{2}[1]$, and thereby $c_{2} \rightarrow c_{2}^{\prime}=\beta_{0} \cdot 0+c_{2}[0]$. This condition transfers 1-loop renormalization of charge, accumulated in the term $a_{s}^{2} \beta_{0} c_{2}[1]$, into the new renormalization scale $\mu^{\prime 2}=$ $\exp \left(-c_{2}[1]\right) \mu^{2}$ of the coupling $a_{s}^{\prime}$, where $\ln \left(\mu^{\prime 2} / \mu^{2}\right)=$ $t^{\prime}-t=-\Delta_{0}$. At the same time, the coefficient $c_{2}^{\prime}$ is reduced to $c_{2}^{\prime}=c_{2}[0]$, the "conformal part" of $c_{2}$. For further generalization of the BLM approach one needs to know about the tracks of charge renormalization in every higher order coefficient $c_{i}$, which are described by the so-called $\{\beta\}$-expansion [8,9] and which are presented in Appendix A.

Another approach is to fit the parameters $\left\{\Delta_{0}, \Delta_{1}\right.$, $\left.\Delta_{2}, \ldots\right\} \equiv\{\boldsymbol{\Delta}\}$ numerically following some criteria of the PT series optimization and ignoring the intrinsic structure of $c_{n}$. One can manage both the values of the PT coefficients $c_{i}^{\prime}(\{\boldsymbol{\Delta}\})$ and the value of the new coupling $a_{s}^{\prime}=\bar{a}_{s}\left(t^{\prime}=t-\Delta\right)$, and thereupon improve the convergence of expansion in the set of Eqs. (7). By the same procedure, by means of Eqs. (5) and (7), we find a way to improve perturbation expansion. The price we pay to achieve this improvement is that we have to control simultaneously both the expansion for $\Delta$ in Eq. (5) and for the coefficients $c_{i}^{\prime}$ in Eq. (7). In the paper, we develop just this approach. In the next section, we will formulate 
the foregoing conditions and discuss the admissible domains of $\left\{\Delta_{0}, \Delta_{1}, \Delta_{2} \ldots\right\}$ following from them.

\section{THE ADMISSIBLE DOMAINS OF $\{\Delta\}$ PARAMETERS}

Let us apply the general scheme of optimization described in the previous section to the relevant quantity $C_{\mathrm{Bjp}}$ starting from the appropriate conditions for the truncated PT series in Eq. (6). At first sight, it might seem that one can choose any value for the new scale $\mu^{\prime}$ and, therefore, the parameters $\left\{\Delta_{0}, \Delta_{1}, \Delta_{2}, \ldots\right\}$ in Eq. (5) might look unconstrained, but that is not true. In order to satisfy the reliability requirements for the PT expansion, we demand natural inequalities (i)-(iii) for its successive terms:

(i) The terms of PT expansion of $\Delta(t)$ in Eq. (5) should be

$\left|\Delta_{0}\right| \geq\left|A^{\prime} \Delta_{1}\right| \geq\left|A^{\prime 2} \Delta_{2}\right|, \quad$ where $A^{\prime} \equiv \beta_{0} a_{s}^{\prime}$,

which means that the next term of this PT expansion cannot be larger than the previous one. These inequalities suppose nonlinear conditions for $\Delta_{i}$, which become more restrictive for the case $\Delta_{0}>0$ by virtue of asymptotic freedom, $A^{\prime} \simeq 1 / t^{\prime}$, where $t^{\prime}$ is defined in Eq. (5).

(ii) For PT expansion in Eq. (6) we impose conditions with respect to $c_{i}^{\prime}$, which are similar to Eq. (8):

$$
1 \geq\left|A^{\prime} \frac{c_{2}^{\prime}}{\beta_{0}}\right| \geq\left|A^{\prime 2} \frac{c_{3}^{\prime}}{\beta_{0}^{2}}\right| \geq\left|A^{\prime 3} \frac{c_{4}^{\prime}}{\beta_{0}^{3}}\right| .
$$

We assume Eqs. (8) and (9) to be necessary conditions, admitting at the same time that we can provide and substantiate more restricted ones. The new coefficients $c_{i}^{\prime}$ are given by Eq. (7), while the explicit forms of the initial coefficients $c_{i}$ are presented in Appendix A. The running $\bar{a}_{s}$ has an asymptotic expansion, Eq. (B8) of Appendix B, or can be taken from the numerical solution of Eq. (B3).

(iii) To fix the PT domain of applicability, we put for the logarithmic variable $t^{\prime}=t-\Delta\left(t^{\prime}\right)$ the appropriate lower bound at $\mu_{0}^{2} \simeq 1 \mathrm{GeV}^{2}$ that corresponds to $t_{\mu_{0}}=\ln \left(\mu_{0}^{2} / \Lambda_{q c d}^{2}\right) \simeq 2.3$ at $\Lambda_{q c d}=\Lambda_{(4)}^{\left(n_{f}=4\right)}=$ $0.318 \mathrm{GeV}$ :

$t, t^{\prime} \geq t_{\mu_{0}} \Rightarrow t-2.3 \geq \Delta\left(t^{\prime}\right)=\Delta_{0}+A^{\prime} \Delta_{1}+A^{\prime 2} \Delta_{2}$.

Next, we will scan $t$ in the practically interesting interval $2.3<t \leq 8\left(1<\mu^{2} \leq 301 \mathrm{GeV}^{2}\right)$ and we will localize at every $t$ the region of the parameters $\left\{\Delta_{0}, \Delta_{1}, \Delta_{2}\right\}$, where the constraint conditions, Eqs. (8), (9), and (10), are fulfilled simultaneously.
These conditions form the admissible domain in the $\{\boldsymbol{\Delta}\}$-space at every value of $t$, denoted as $\{\overline{\boldsymbol{\Delta}}\}$, where one can perform optimization.

\section{A. 2D optimization}

We consider first $C_{\mathrm{Bjp}}$, Eq. (6), of order $O\left(a_{s}^{3}\right)$ for twodimensional parametrization, $\left\{\Delta_{0}, \Delta_{1}\right\}$. In this case, the conditions, Eqs. (8), (9), and (10), obtain the shortened form

$$
\begin{gathered}
\left|\Delta_{0}\right| \geq\left|A^{\prime} \Delta_{1}\right|, \\
1 \geq\left|A^{\prime} \frac{c_{2}^{\prime}}{\beta_{0}}\right| \geq\left|A^{\prime 2} \frac{c_{3}^{\prime}}{\beta_{0}^{2}}\right|, \\
t \geq t_{\mu_{0}}+\Delta_{0}+A^{\prime} \Delta_{1} .
\end{gathered}
$$

The corresponding admissible domains calculated numerically for $t=3,4, \ldots, 8$ or, respectively, for $\mu^{2}=2.0,5.5$, 15.0, 40.8, 111, $301 \mathrm{GeV}^{2}$ at $\Lambda_{(4 \text { loop })}^{\left(n_{f}=4\right)}=0.318 \mathrm{GeV}$ are shown in Fig. 1. The constraints in Eqs. (11b) and (11c) are much more restrictive for the parameters in the right half plane for $\Delta_{0}>0$. Therefore, the corresponding domains are significantly smaller than in the left half plane, where $\Delta_{0}<0$. It is worth noting that the "standard" BLM value, $\left\{\Delta_{0}=2, \Delta_{i}=0\right\}$, [7] also belongs to the admissible domain. Moreover, the larger $t$ is, the larger the corresponding admissible domain that is the manifestation of

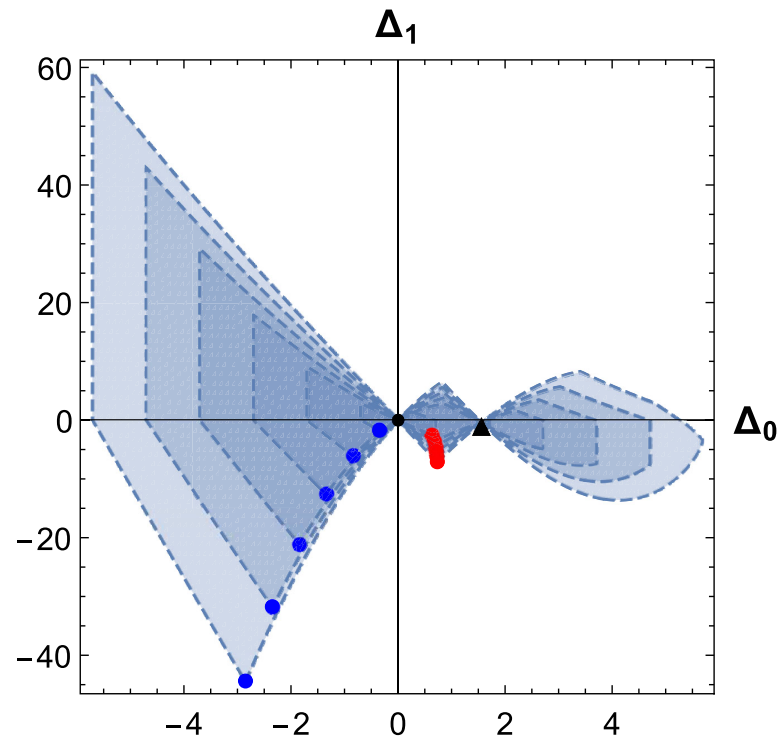

FIG. 1. 2D domains for admissible parameters $\left\{\bar{\Delta}_{0}, \bar{\Delta}_{1}\right\}$ at different $t$, distinguished by different degrees of gray: $t=3$ (dark), $\ldots, t=8$ (light). The black triangle on the right half plane corresponds to the conditions $c_{2}^{\prime}=c_{3}^{\prime}=0$. Blue points (on the left) and red points (on the right) are the bare (global) and the constrained (local) minima of the radiative corrections, respectively. 

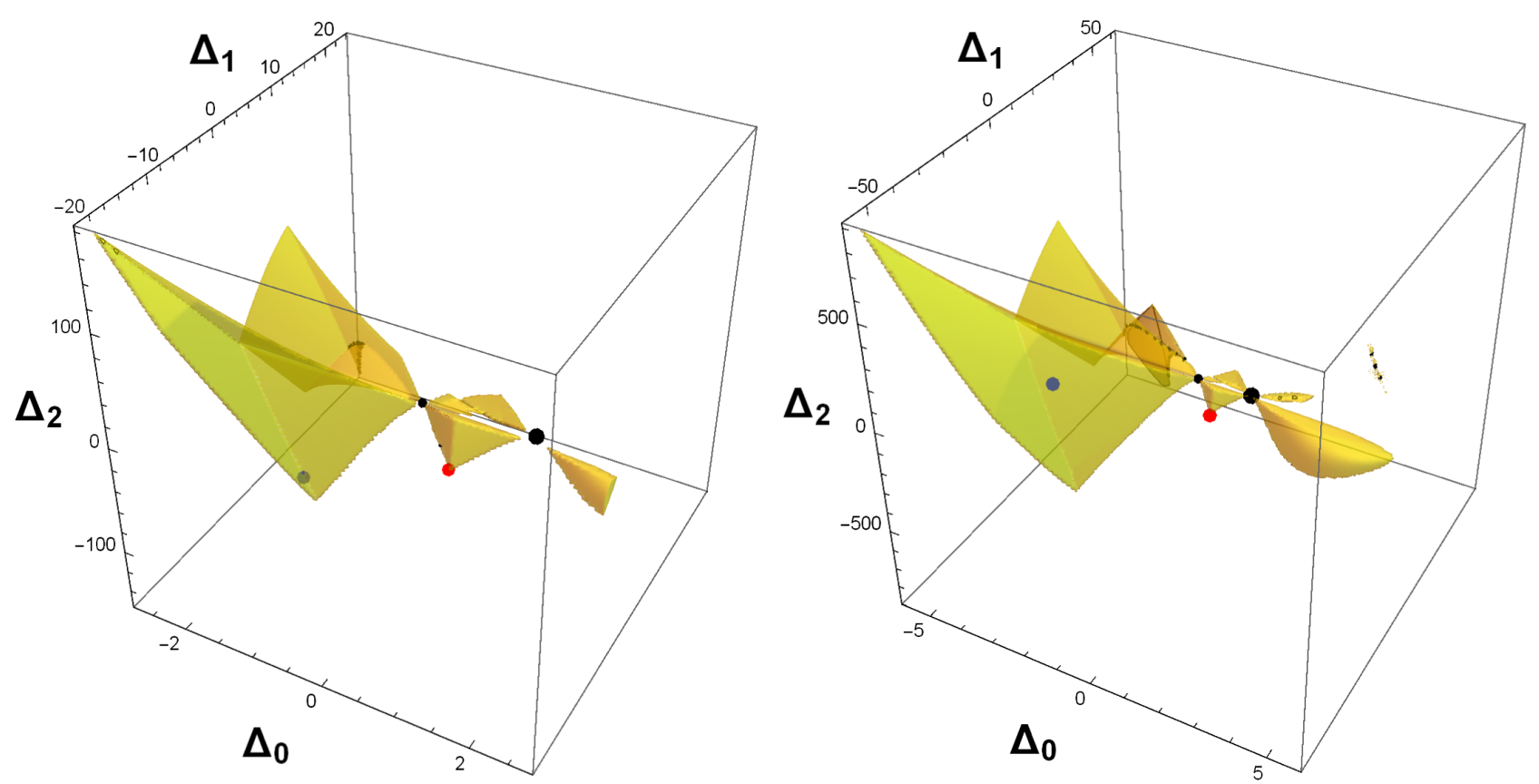

FIG. 2. 3D domains for admissible parameters $\left\{\bar{\Delta}_{0}, \bar{\Delta}_{1}, \bar{\Delta}_{2}\right\}$ at $t=5$ (left) and $t=8$ (right). The large black ball on the right half plane corresponding to the conditions $c_{2}^{\prime}=c_{3}^{\prime}=c_{4}^{\prime}=0$ does not belong to the admissible region for both the cases. Blue $\left(\Delta_{0}<0\right)$ and red $\left(\Delta_{0}>0\right)$ points within the domains denote the bare (global) and the constrained (local) minima of the radiative corrections, respectively, similarly as in Fig. 1.

asymptotic freedom. The point $(0,0)$ in the $\left(\Delta_{0}, \Delta_{1}\right)$-plane in Fig. 1 corresponds to the nonoptimized result of $C_{\mathrm{Bjp}}$, Eq. (2a), while the points corresponding to the optimized one, Eq. (6), are depicted for both $\Delta_{0}<0$ (left, blue circles) and $\Delta_{0}>0$ (right, red circles). The black triangle, also lying in the admissible area, represents the conditions $c_{2}^{\prime}=c_{3}^{\prime}=0$ studied in [9],

$$
\begin{aligned}
c_{2}^{\prime} & =c_{3}^{\prime}=0 \Rightarrow \Delta_{0}=c_{2} / \beta_{0}=1.56, \\
\Delta_{1} & =\left(c_{3}-c_{2}^{2}-c_{2} \beta_{1} / \beta_{0}\right) / \beta_{0}^{2} \approx-0.396 .
\end{aligned}
$$

The conditions in Eqs. (12) correspond to the new norm scale $\mu^{\prime 2}=\mu^{2} \exp \left[-\Delta\left(a_{s}^{\prime}\right)=-1.56+0.396 \beta_{0} a_{s}\left(\mu^{\prime 2}\right)\right]>$ $0.22 \mu^{2}$; see Fig. 1 (right) in [9]. If one imposes also the fourth term in the condition (ii), Eq. (9), for the $2 \mathrm{D}$ parametrization, $\left\{\Delta_{0}, \Delta_{1}\right\}$, the domains $\{\overline{\boldsymbol{\Delta}}\}$ in the right half plane become slightly disintegrated and get a cut along the $\Delta_{0}$ direction. This effect can be seen on the corresponding cross section at $\Delta_{2}=0$ of $3 \mathrm{D}$ admissible domains presented in Fig. 2.

\section{B. 3D optimization}

Next we consider the admissible $3 \mathrm{D}$ domains $\left\{\bar{\Delta}_{0}, \bar{\Delta}_{1}\right.$, $\left.\bar{\Delta}_{2}\right\}$ in the order of $O\left(a_{s}^{4}\right)$. Examples for $t=5$ $\left(\mu^{2} \approx 15.0 \mathrm{GeV}^{2}\right)$ and $t=8\left(\mu^{2} \approx 301.0 \mathrm{GeV}^{2}\right)$ are shown in Fig. 2 in the left and right panels, respectively. We can see again that the larger $t$ is, the larger the corresponding admissible domain. In comparison to the 2D case, 3D admissible domains become disintegrated for $\Delta_{0}>0$, having a cut along the $\Delta_{0}$ direction. Moreover, the large black balls in the $3 \mathrm{D}$ plots corresponding to the conditions $c_{2}^{\prime}=c_{3}^{\prime}=c_{4}^{\prime}=0$, an analogue of the $2 \mathrm{D}$ condition, Eq. (12), are not contained in the admissible regions. The reason is obvious: this condition contradicts the inequality, Eq. (8), for the perturbation expansion of $\boldsymbol{\Delta}$ for $t \leq 9$.

\section{THE RESULTS OF RENORMALIZATION GROUP OPTIMIZATION FOR BSR}

In the previous sections, we have generally described the method of RG optimization. Now we present the numerical results of this optimization for the Bjorken sum rule. We consider optimization of c.f. $C_{\mathrm{Bjp}}$ as a determination of the minimum of radiative corrections by varying the parameters $\left\{\Delta_{0}, \Delta_{1}, \Delta_{2}\right\} .{ }^{2}$ We find numerically the minimum of the function $\left|f_{\text {Rad }}\right|$ which accumulates all of the known radiative corrections up to order $\alpha_{s}^{4}$,

$$
\begin{array}{r}
C_{\mathrm{Bjp}}\left(t^{\prime}, a_{s}^{\prime}\right)=1+c_{1} f_{\mathrm{Rad}}(t ;\{\boldsymbol{\Delta}\}) \equiv 1-\alpha_{s}^{g_{1}} / \pi, \\
\left.f_{\mathrm{Rad}}(t ; \boldsymbol{\Delta}\}\right)=a_{s}^{\prime}\left(1+a_{s}^{\prime} c_{2}^{\prime}+\left(a_{s}^{\prime}\right)^{2} c_{3}^{\prime}+\left(a_{s}^{\prime}\right)^{3} c_{4}^{\prime}\right) .
\end{array}
$$

\footnotetext{
${ }^{2}$ The problem of optimization of QCD radiative corrections in the parameter space $\{\boldsymbol{\Delta}\}$ was formulated in [9].
} 
The quantity $\left|c_{1} f_{\operatorname{Rad}}(t ;\{0\})\right|=4 a_{s}\left(1+a_{s} c_{2}+\left(a_{s}\right)^{2} c_{3}+\right.$ $\left.\left(a_{s}\right)^{3} c_{4}\right)$ is the auxiliary "effective $\alpha_{s}^{g_{1}} / \pi$ " charge proposed in $[10,16]$. The arguments $\{\boldsymbol{\Delta}\}$ of $\left.f_{\mathrm{Rad}}(t ; \boldsymbol{\Delta}\}\right)$ are taken within the admissible domain $\{\overline{\boldsymbol{\Delta}}\}$ at every $t$. The approach of the PT optimization when the bare (global) minimum of $\mid f_{\operatorname{Rad}}(t ;\{\boldsymbol{\Delta}\} \mid$ is restricted by a set of inequality constraints, Eqs. (8)-(10), is universal and does not depend on the knowledge of details of the $\{\beta\}$ expansion for the quantity. Indeed, in our numerical analysis, we will not need any information about the intrinsic structure of the PT coefficients $c_{i}$.

\section{A. Numerical results of the renormalization group optimization of $\boldsymbol{C}_{\mathbf{B} \mathbf{j p}}$}

We compare different optimization results with the initial nonoptimized one, Eq. (3a),

$$
\begin{aligned}
\left\{\Delta_{0}=\right. & \left.\Delta_{1}=\ldots=0\right\} \\
C_{\mathrm{Bjp}}\left(1, a_{s}\left(m_{\tau}^{2}\right)\right)= & 1-4(0.0264+0.0090 \\
& +0.0041+0.0032+\ldots) \\
= & 1-4(0.0428)=1-\mathbf{0 . 1 7 1}=1-\alpha_{s}^{g_{1}} / \pi .
\end{aligned}
$$

Let us start with the 2D optimization in the $\left\{\Delta_{0}, \Delta_{1}\right\}$-space. The optimal points (blue circles in Fig. 1) are located for $\Delta_{0}<0$ on the boundary of admissible domains $\left\{\bar{\Delta}_{0}, \bar{\Delta}_{1}\right\}$. For $t_{\tau}=\ln \left(m_{\tau}^{2} / \Lambda_{q c d}^{2}\right) \approx 3.44$ and $t_{\tau}^{\prime}=t_{\tau}-\Delta$ we have

$$
\begin{aligned}
\left\{\Delta_{0}=\right. & \left.-0.571, \Delta_{1}=-3.35, \Delta_{2}=0\right\} \\
C_{\mathrm{opt}, 1}^{\Delta}\left(a_{s}^{\prime}\right) \equiv & C_{\mathrm{Bjp}}\left(t_{\tau}^{\prime}, a_{s}^{\prime}\right) \\
= & 1-4(0.0205+0.0074 \\
& +0.0054+0.0038+\ldots) \\
= & 1-4(0.0371) \\
= & 1-\mathbf{0 . 1 4 9}=1-\alpha_{s, \mathrm{opt} 1}^{g_{1}} / \pi .
\end{aligned}
$$

From the comparison between two cases: Eqs. (14) and (15c), we can see that the effective $\alpha_{s}^{g_{1}} / \pi$ changes from $\mathbf{0 . 1 7 1}$ to $\mathbf{0 . 1 4 9}$, respectively, giving $\delta_{\text {opt } 1} \equiv \alpha_{s}^{g_{1}} / \pi-\alpha_{s, \mathrm{opt} 1}^{g_{1}} / \pi \approx$ 0.023 . The advantage of the optimized result looks substantial, while the values of the shift $\Delta$ in Eq. (15a) are moderate.

The next step is to find the minimum of $\left|f_{\text {Rad }}\right|$ at the additional condition, $\Delta_{0}>0$. This corresponds with the original BLM result, $\Delta_{0}^{\mathrm{BLM}}=2$ [see Eq. (A3b)], and also with similar results within the PMC [10]. The corresponding minima positions for $\Delta_{0}>0$, depicted as red circles in Fig. 1, provide the following estimation:

$$
\Delta_{0}>0 \quad\left\{\Delta_{0}=0.660, \Delta_{1}=-2.98, \Delta_{2}=0\right\}
$$

$$
\begin{aligned}
C_{\mathrm{opt}, 2}^{\Delta}\left(a_{s}^{\prime}\right) \equiv & C_{\mathrm{Bjp}}\left(t_{\tau}^{\prime}, a_{s}^{\prime}\right) \\
= & 1-4(0.0266+\underline{0.0053} \\
& +\underline{0.0053}+0.0038+\ldots) \\
= & 1-4(0.0410)=1-0.164 .
\end{aligned}
$$

This "optimum" result in Eq. (16c) is not pronounced in comparison with Eq. (15c). At the same time, this solution appears on the boundary, where $\left(a^{\prime}\right)^{2} c_{2}^{\prime} \approx\left(a^{\prime}\right)^{3} c_{3}^{\prime}$; see the underlined terms. This makes PT convergence worse and the final result less reliable.

For a similar 3D optimization within the admissible domains shown in Fig. 2 we obtain

$$
\begin{aligned}
\left\{\Delta_{0}=\right. & \left.-0.381, \Delta_{1}=-2.21, \Delta_{2}=-12.8\right\} \\
C_{\mathrm{opt}, 3}^{\Delta}\left(a_{s}^{\prime}\right) \equiv & C_{\mathrm{Bjp}}\left(t_{\tau}^{\prime}, a_{s}^{\prime}\right) \\
= & 1-4(0.0207+0.0069 \\
& +\underline{0.0043}+\underline{0.0042}+\ldots) \\
= & 1-4(0.0361)=1-0.144=1-\alpha_{s, \mathrm{opt} 3}^{g_{1}} / \pi
\end{aligned}
$$

and

$$
\begin{aligned}
\Delta_{0}>0\left\{\Delta_{0}=\right. & \left.0.573, \Delta_{1}=-2.72, \Delta_{2}=-5.69\right\} \\
C_{\mathrm{opt}, 4}^{\Delta}\left(a_{s}^{\prime}\right) \equiv & C_{\mathrm{Bjp}}\left(t_{\tau}^{\prime}, a_{s}^{\prime}\right) \\
= & 1-4(0.0253+0.0053 \\
& +\underline{0.0045}+\underline{0.0044}+\ldots) \\
= & 1-4(0.0396)=1-0.158 .
\end{aligned}
$$

We see that the 3D analysis has no significant advantages over the corresponding 2D results, Eqs. (15c) and (16c). The underlined terms in Eqs. (17b) and (18b) illustrate that the PT convergence is not good enough to make the results reliable. Summarizing our tests among the considered cases, only the $2 \mathrm{D}$ result, Eq. (15c), is at a near optimum level providing satisfactory convergence of the PT series.

Let us comment on the disadvantage of the used numerical approach. Namely, "blind analysis" based on the constraints, Eqs. (8)-(10), can lead to the unsatisfactory solution. Indeed, the minima of the radiative corrections in the cases, Eqs. (16b), (17b), and (18b), occur on the boundary of the constraint, Eq. (9) (see the underlined terms), where the PT convergence deteriorates. Another lesson from these numerical tests is that the scales of "BLM/PMC," corresponding to the additional condition $\Delta_{0}>0$, lead to the constrained (local) minimum which is not close to the global minimum of radiative corrections. 
In this connection, it is instructive to compare the results for $\alpha_{s \mathrm{PMC}}^{g_{1}}\left(Q^{2}\right) / \pi$ obtained in [10] within the PMC scale setting with our predictions. The PMC is based on some version of $\beta$-expansion ${ }^{3}$ and the assumption about sufficient convergence of the truncated PT series. The conventional pQCD effective charge $\alpha_{s}^{g_{1}} / \pi>\alpha_{s \mathrm{PMC}}^{g_{1}} / \pi$ at much better convergence of the latter. Let us briefly discuss similarities: (i) The PMC scales qualitatively agree with the scales $\Delta$ obtained in this paper for the local minimum $\Delta_{0}>0$ in Eqs. (16a) and (18a). (ii) The advantage of the PMC defined as $\delta_{\mathrm{PMC}}=\left.\left(\alpha_{s}^{g_{1}} / \pi-\alpha_{\mathrm{sPMC}}^{g_{1}} / \pi\right)\right|_{Q^{2}=m_{\tau}^{2}} \approx 0.013$ (see Fig. 1 in [10]) is numerically close to $\delta$ from Eq. (18c) here. In contrast, our 2D optimization for the global minimum, Eq. (15), gives an almost twice higher advantage with appropriate convergence, $\delta_{\text {opt1 }} \approx 0.023 \mathrm{vs} \delta_{\text {PMC }}$. These differences originate from the fact that in our approach the optimization is tightly related to the global minimum of the partial sum of radiative corrections, as opposed to the PMC purpose of faster convergence.

\section{B. Optimized Bjorken sum rule vs different experimental results}

In this subsection, we compare the results for the BSR obtained here for the optimized PT series for the leading twist contributions with the experimental measurements of COMPASS [18-20] (taking into account the results of truncated moments [11,12,21]), the E155 Collaboration at SLAC [22], and JLab EG1-DVCS [23]. These optimized estimates are expectedly higher than the conventional ones.

\section{Optimized Bjorken sum rule for COMPASS measurements}

Experimental verification of the DIS sum rules always encounters the difficulty that in any realistic experiment one cannot reach arbitrarily small values of the Bjorken $x$, $x \geq x_{0} \equiv Q_{\min }^{2} /\left(2(P q)_{\max }>0\right)$, where $P$ is hadronic momentum and $q$ the momentum transfer of the DIS. The method of truncated Mellin moments (TMM) operating in the range $\left(x_{0}, 1\right)$ can overcome this $x_{0}$ problem [24-26]. To obtain the optimized phenomenological result for BSR, $\Gamma_{1}^{\exp } \simeq \Gamma_{1}\left(x_{0}\right)=\int_{x_{0}}^{1} g_{1}^{(n s)}\left(x ; \mu^{2}\right) d x$, we used the tBSR approach which incorporates experimental uncertainties on the spin function $g_{1}[12,21]$. The tBSR elaborated in [11] is based on the TMM approach providing not only a natural framework of DIS analysis in the restricted kinematic region of $x \geq x_{0}$ but also allowing an effective study of the sum rules in a low $x$ limit. Since the tBSR saturates in the low- $x$ limit much sooner than the ordinary BSR [11], we assume a smaller systematic error and the

\footnotetext{
${ }^{3}$ Let us mention here that we do not agree with a certain construction of $\{\beta\}$-expansion used in [10] for $c_{2,3,4}$; see our criticism in $[9,17]$.
}

total one of the level of 5\% at a conservative approach to this estimation. Thus, we find for the COMPASS data

$$
\Gamma_{1(c-s s)}^{\mathrm{exp}-\mathrm{opt}}=0.191 \pm 0.01
$$

which is in good agreement with the most recent COMPASS result provided for $Q^{2}=3 \mathrm{GeV}^{2}$ [20]:

$$
\Gamma_{1(c-s s)}^{\exp }=0.192 \pm 0.007_{\text {stat }} \pm 0.015_{\text {syst }} \text {. }
$$

In the previous section, we discussed the optimized results for the QCD radiative corrections at the world reference scale $m_{\tau}^{2}$. Below, we provide similar results starting with $C_{\mathrm{Bjp}}\left(1, a_{s}\left(Q^{2}\right)\right)$ at the COMPASS reference scale $Q^{2}=3 \mathrm{GeV}^{2}$. Thus, we obtain

$$
\begin{aligned}
& C_{\mathrm{Bjp}}\left(1, a_{s}\left(Q^{2}\right)\right) \\
& \quad=1-4(0.0268+0.0093+0.0043+0.0034+\ldots) \\
& \quad=1-4(0.0438)=1-0.175 .
\end{aligned}
$$

Then, using the already discussed and approved 2D $\left\{\Delta_{0}, \Delta_{1}\right\}$ optimization in Eq. (15), we find the optimized value of $C_{\mathrm{Bjp}}$,

$$
\begin{aligned}
& \left\{\Delta_{0}=-0.545, \Delta_{1}=-3.13, \Delta_{2}=0\right\} \\
& C_{\mathrm{opt}}^{\Delta}\left(a_{s}^{\prime}\right) \equiv C_{\mathrm{Bjp}}\left(t^{\prime}, a_{s}^{\prime}\right) \\
& =1-4(0.0209+0.0077+0.0055+0.0039+\ldots)
\end{aligned}
$$

$$
=1-4(0.0380)=1-0.152,
$$

that is visibly larger than the nonoptimized result $1-0.175$ in Eq. (21). These values lead to the following estimates for the leading twist-2 part of $\Gamma_{1}^{\text {th }}$ in Eq. (1):

$$
\begin{aligned}
\Gamma_{1, t w 2}^{\text {th-non-opt }}\left(Q^{2}\right) & =\left|\frac{g_{A}}{6}\right|_{\mathrm{C}-\mathrm{SS}} C_{\mathrm{Bjp}}\left(1, a_{s}\left(Q^{2}\right)\right) \\
& \approx 1.29 / 6 \cdot 0.825=0.177 \pm 0.003, \\
\Gamma_{1, t w 2}^{\mathrm{th}-\mathrm{opt}}\left(Q^{2}\right) & =\left|\frac{g_{A}}{6}\right|_{\mathrm{C}-\mathrm{SS}} C_{\mathrm{opt}}^{\Delta}\left(a_{s}^{\prime}\right) \approx 1.29 / 6 \cdot 0.848 \\
& =0.182 \pm 0.003,
\end{aligned}
$$

where $\left|g_{A}\right|_{\text {C-SS }}=1.29 \pm 0.05_{\text {stat }} \pm 0.1_{\text {syst }}$ is the specific estimate obtained together with $\Gamma_{1(c-s s)}^{\exp }$ in Eq. (20) in the recent COMPASS measurement [20]. The uncertainties of $\Gamma_{1, t w 2}^{\mathrm{th}}$ are determined by the uncertainty of $\alpha_{s}\left(m_{\tau}^{2}\right)$. The result of the 3D optimization for the bare minimum, $\Gamma_{1, t w 2}^{\mathrm{th}} \approx 0.183$, does not improve the estimate distinctly. 
It is seen from the comparison of the nonoptimized result, Eq. (23a); the optimized one, Eq. (23b); and then the prediction of the tBSR approach, Eq. (19), with the experimental result, Eq. (20), that the optimization reduces the differences between theoretical and experimental (exp, exp-opt) estimations.

Let us now briefly discuss possible implementation of HT corrections in our theoretical analysis. At lower $Q^{2}$ the HT power corrections $\mu_{4}^{p-n} / Q^{2}, \mu_{6}^{p-n} / Q^{4}$, etc., Eq. (1), are needed to describe the data. The precise JLab data on $\Gamma_{1}^{p-n}\left(Q^{2}\right)$ at low $Q^{2}$ provided a good test for both the perturbative leading-twist (LT) and nonperturbative HT contributions. Several theoretical and experimental estimates of the scales $\mu_{2 i}$ have been made showing that impact of the HT corrections can be important for $Q^{2} \lesssim 5 \mathrm{GeV}^{2}$; see, e.g., [23,27-32]. After taking into account the first HT term $\mu_{4}^{p-n}$, which is negative, the difference between our theoretical prediction and the COMPASS results will increase even more. For the estimate $\mu_{4(k m t k)}^{p-n} / M^{2}=$ $-0.047 \pm 0.02$ from [11], we obtain

$\Gamma_{1}^{\text {th-opt }}\left(Q^{2}\right)=\Gamma_{1, t w 2}^{\text {th-opt }}\left(Q^{2}\right)+\frac{\mu_{4(k m t k)}^{p-n}}{Q^{2}}=0.167 \pm 0.007$,

while for the experimental estimate provided by JLab EG1-DVCS [23], $\mu_{4(\mathrm{JLab})}^{p-n} / M^{2}=-0.021 \pm 0.016$, we have

$\Gamma_{1}^{\mathrm{th}-\mathrm{opt}}\left(Q^{2}\right)=\Gamma_{1, t w 2}^{\mathrm{th}-\mathrm{opt}}\left(Q^{2}\right)+\frac{\mu_{4(\mathrm{JLab})}^{p-n}}{Q^{2}}=0.175 \pm 0.006$,

where the uncertainty of $\Gamma_{1}^{\text {th-opt }}$ is the combined uncertainty from $\alpha_{s}$ and HT. This latter result in Eq. (25) is supported by the COMPASS value $\Gamma_{1(c-s s)}^{\exp }$ in Eq. (20) within combined statistical and systematic uncertainty and does not contradict even more restricted $\Gamma_{1(c-s s)}^{\text {exp -opt }}$ in Eq. (19).

\section{Optimized Bjorken sum rule for SLAC E155 Collaboration measurements}

The initial result for the coefficient function $C_{\mathrm{Bjp}}$ at the reference scale $Q^{2}=5 \mathrm{GeV}^{2}$ of E155 measurements reads

$$
\begin{aligned}
C_{\mathrm{Bjp}}\left(1, a_{s}\left(Q^{2}\right)\right)= & 1-4(0.0236+0.0073 \\
& +0.0029+0.0020+\ldots) \\
= & 1-4(0.0358)=1-0.143=0.857 .
\end{aligned}
$$

Within the 2D $\left\{\Delta_{0}, \Delta_{1}\right\}$ optimization, Eq. (26), at $Q^{2}$ we find

$$
\left\{\Delta_{0}=-0.800, \Delta_{1}=-5.513, \Delta_{2}=0\right\}
$$

$$
\begin{aligned}
C_{\mathrm{opt}}^{\Delta}\left(a_{s}^{\prime}\right) \equiv C_{\mathrm{Bjp}}\left(t^{\prime}, a_{s}^{\prime}\right) \\
=1-4(0.0174+0.0060+0.0046+0.0029+\ldots) \\
\\
=1-4(0.0309)=1-0.124=0.876, \quad(27 \mathrm{c})
\end{aligned}
$$

while within the $3 \mathrm{D}$ optimization we obtain the result that is very close to the previous one but with badly convergent PT series:

$$
\begin{aligned}
\left\{\Delta_{0}\right. & \left.=-0.548, \Delta_{1}=-3.58, \Delta_{2}=-24.3\right\} \\
C_{\mathrm{opt}, 5}^{\Delta}\left(a_{s}^{\prime}\right) & \equiv C_{\mathrm{Bjp}}\left(t_{\tau}^{\prime}, a_{s}^{\prime}\right) \\
& =1-4(0.0177+0.0055+\underline{0.0035}+\underline{0.0035}+\ldots) \\
& =1-4(0.0301)=1-0.120=0.880 .
\end{aligned}
$$

Therefore, hereafter we take the 2D result, Eq. (27c), which gives the leading-twist contribution of BSR,

$$
\begin{aligned}
\Gamma_{1, t w 2}^{\mathrm{th}-\mathrm{opt}}\left(Q^{2}\right) & =\left|\frac{g_{A}}{6}\right| C_{\mathrm{opt}}^{\Delta}\left(a_{s}^{\prime}\right) \\
& =1.27 / 6 \cdot 0.876=0.186 \pm 0.002,
\end{aligned}
$$

where $\left|g_{A}\right|=1.2723 \pm 0.0023$ [33]. Including in the estimates of Eq. (1) also the HT contributions with the values $\mu_{4(k m t k)}^{p-n} / M^{2}[11]$ and $\mu_{4(\text { JLab })}^{p-n} / M^{2}$ [23], we obtain

$\Gamma_{1}^{\mathrm{th}-1}\left(Q^{2}\right)=\Gamma_{1, t w 2}^{\mathrm{th}-\mathrm{opt}}\left(Q^{2}\right)+\frac{\mu_{4(k m t k)}^{p-n}}{Q^{2}}=0.177 \pm 0.004$,

$\Gamma_{1}^{\mathrm{th}-2}\left(Q^{2}\right)=\Gamma_{1, t w 2}^{\mathrm{th}-\mathrm{ppt}}\left(Q^{2}\right)+\frac{\mu_{4(\mathrm{JLab})}^{p-n}}{Q^{2}}=0.182 \pm 0.003$.

The optimized estimation in Eq. (30a) is in good agreement with the experimental result

$$
\Gamma_{1(\mathrm{SLAC})}^{\exp }=0.176 \pm 0.003_{\mathrm{stat}} \pm 0.007_{\mathrm{sys}},
$$

while for the smaller in modulo HT correction in Eq. (30b) agreement to the data is reasonable.

\section{Optimized Bjorken sum rule for JLab EG1-DVCS measurements}

It is worthwhile to compare our analysis with the recent high precision determination of BSR at JLab [23]. To this aim, we choose JLab EG1-DVCS data covering the range $1.0 \leq Q^{2} \leq 4.8 \mathrm{GeV}^{2}$ where the perturbative methods are justified. These experimental results are compared with the optimized predictions of BSR in Fig. 3. 


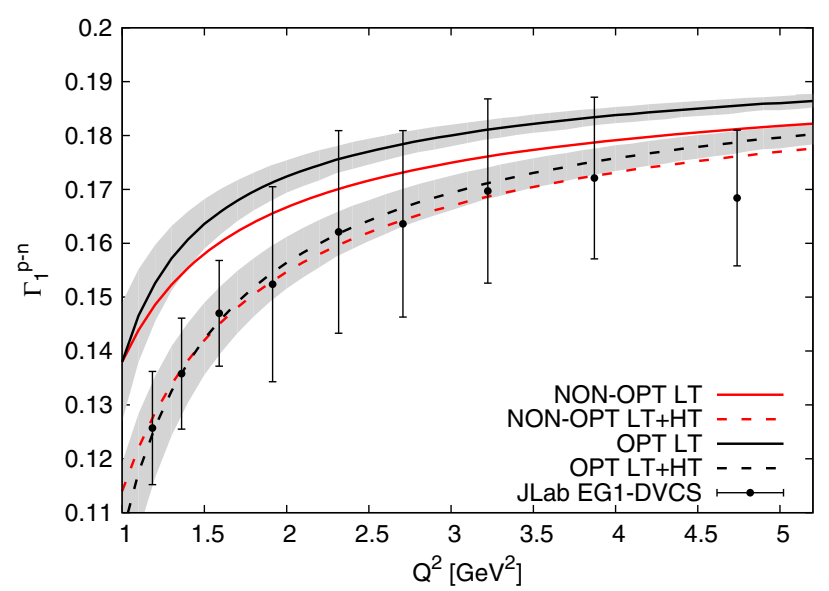

FIG. 3. Comparison of the optimized (black solid curve) and nonoptimized (red solid lower curve) predictions on BSR with the experimental EG1-DVCS data. The impact of the twist-4 correction is also shown (dashed). For better visibility we show the error band only for the optimized plots.

We use twist-2 optimized values, Eqs. (1) and (13b), calculated for different experimental $Q^{2}>1 \mathrm{GeV}^{2}$ together with the nonoptimized ones, Eqs. (1) and (2b). For both cases we also estimated twist- 4 correction from the data and obtained $\mu_{4}^{p-n} / M^{2}=-0.034 \pm 0.007$ for the optimized and $-0.026 \pm 0.007$ for the nonoptimized approach. Figure 3 shows that the pure LT contribution to BSR lies significantly above the experimental data for both kinds of theoretical results, and the difference grows with decreasing $Q^{2}$, motivating the necessity of HT corrections. It is found that the value $\mu_{4(\mathrm{opt})}^{p-n} / M^{2}=-0.034 \pm 0.007$ estimated from comparison of the optimized predictions to the data is compatible with the experimental value provided by JLab EG1-DVCS and also with other theoretical estimations. It is also seen that the optimized approach to the value LT + HT describes well the $Q^{2}$ evolution of BSR even down to small $Q$ values.

\section{CONCLUSIONS}

We have discussed a possible improvement in theoretical determination of the polarized Bjorken sum rule $\Gamma_{1}^{p-n}$. We performed minimization of the partial sums of the QCD perturbation series for the coefficient function $C_{\mathrm{Bjp}}\left(Q^{2} / \mu^{2}, \alpha_{s}\left(\mu^{2}\right)\right)$ of the leading twist for a certain DIS process by means of an appropriate normalization scale $\mu^{2}$ resulting from the renormalization group. To this aim, we provided a set of general conditions for the optimized analysis of $C_{\mathrm{Bjp}}$ within the perturbation QCD and the renormalization group. This frame is universal and applicable for the analysis of any renormalization group invariant quantities. Based on these conditions, we found the admissible domains for the corresponding new normalization scales $\mu^{2}$ for the cases of QCD corrections of the orders of $O\left(\alpha_{s}^{3}\right)$ and $O\left(\alpha_{s}^{4}\right)$. For these domains we found numerically the minimum of the radiative corrections to $C_{\mathrm{Bjp}}\left(\alpha_{s}\right)$ based on the 4-loop run of $\alpha_{s}\left(\mu^{2}\right)$. This leads to the optimum values of the theoretical predictions for BSR. The optimized results for BSR in the order $O\left(\alpha_{s}^{4}\right)$ are systematically higher than the standard ones and the difference varies between 0.006 at $Q^{2}=2 \mathrm{GeV}^{2}$ and 0.003 at $Q^{2}=10 \mathrm{GeV}^{2}$. We compared these optimized results including also the essential twist-4 correction with the experimental measurements of COMPASS [20], E155 [22], and JLab EG1-DVCS [23]. We obtained for COMPASS $\Gamma_{1}^{\text {th-opt }}\left(3 \mathrm{GeV}^{2}\right)=0.175 \pm 0.006$ and for E155 $\operatorname{SLAC} \Gamma_{1}^{\text {th-opt }}\left(5 \mathrm{GeV}^{2}\right)=0.177 \pm 0.004$. Thus, for the precise E155 data we obtained good agreement with the experimental value $\Gamma_{1(\text { SLAC })}^{\exp }=0.176 \pm 0.003_{\text {stat }} \pm 0.007_{\text {sys }}$ while for COMPASS data, which suffer from large statistical and experimental systematical uncertainties compared to the SLAC E155 or JLab EG1-DVCS results, we obtained reasonable agreement within the combined statistical and systematic uncertainty. From comparison with the EG1-DVCS precise data for $Q^{2}>1 \mathrm{GeV}^{2}$ we found that the optimized approach to LT + HT describes well the $Q^{2}$ evolution of BSR even down to small $Q$ values. Comparing the optimized predictions to the JLab data, we estimated the twist- 4 correction and obtained $\mu_{4(\mathrm{opt})}^{p-n} / M^{2}=$ $-0.034 \pm 0.007$ which is compatible with the experimental value $\mu_{4(\mathrm{JLab})}^{p-n} / M^{2}=-0.021 \pm 0.016$ provided by EG1DVCS and also with other theoretical estimations.

\section{ACKNOWLEDGMENTS}

We would like to thank A. L. Kataev and O. V. Teryaev for the fruitful discussions and Y. Bedfer and E.M. Kabus for the communication of the data. D. K. thanks A. Kotlorz for his help in numerical computations. This work is supported by the Bogoliubov-Infeld Program. D. K. acknowledges the support of the Collaboration Program JINR-Bulgaria 2019. S. V. M. acknowledges the support of the JINR-BelRFFR, Grant No. F18D-002.

\section{APPENDIX A: $\{\beta\}$-EXPANSION FOR $C_{\mathrm{B} \text { jp }}$}

1. The $\{\beta\}$-expansion representation introduced in [8] prescribes the following form of decomposition of the perturbation coefficients $c_{n}$ for $C_{\mathrm{Bjp}}$ in Eq. (2) or for any other RGI quantities: 


$$
\begin{aligned}
c_{1}= & c_{1}[0] \\
c_{2}= & \beta_{0} c_{2}[1]+c_{2}[0] \\
c_{3}= & \beta_{0}^{2} c_{3}[2]+\beta_{1} c_{3}[0,1]+\beta_{0} c_{3}[1]+c_{3}[0] \\
c_{4}= & \beta_{0}^{3} c_{4}[3]+\beta_{1} \beta_{0} c_{4}[1,1]+\beta_{2} c_{4}[0,0,1]+\beta_{0}^{2} c_{4}[2]+\beta_{1} c_{4}[0,1]+\beta_{0} c_{4}[1] \\
& +c_{4}[0] \\
& \vdots \\
c_{n}= & \beta_{0}^{n-1} c_{n}[n-1]+\cdots+c_{n}[0]
\end{aligned}
$$

where $\beta_{i}$ are the expansion coefficients of the QCD $\beta$-function presented in Appendix B,

$$
\mu^{2} \frac{d a_{s}\left(\mu^{2}\right)}{d \mu^{2}}=\beta\left(a_{s}\right)=-a_{s}^{2}\left(\mu^{2}\right) \sum_{i \geq 1} \beta_{i-1} a_{s}^{i-1}\left(\mu^{2}\right) .
$$

The decomposition in Eqs. (A1) contains complete knowledge about $\alpha_{s}$-renormalization in each order of expansion for the RGI quantity $C_{\mathrm{Bjp}}$. It makes it possible to work on optimization of the perturbation series. According to Eq. (A1), the explicit form of the $\{\beta\}$-expansion for $C_{\mathrm{Bjp}}$ within the sequential BLM approach [9] is

$$
\begin{aligned}
C_{\mathrm{Bjp}}\left(a_{s}\right)= & 1+a_{s}\left(-3 C_{F}\right) \\
& +a_{s}^{2}\left(-3 C_{F}\right) \cdot\left\{\frac{1}{3} \mathrm{C}_{\mathrm{A}}-\frac{7}{2} \mathrm{C}_{\mathrm{F}}+2 \cdot \beta_{0}\right\} \\
& +a_{s}^{3}\left(-3 C_{F}\right) \cdot\left\{\frac{115}{18} \cdot \beta_{0}^{2}+\left(\frac{59}{12}-4 \zeta_{3}\right) \cdot \beta_{1}\right. \\
& -\left[\left(\frac{215}{36}-32 \zeta_{3}+\frac{40}{3} \zeta_{5}\right) \mathrm{C}_{\mathrm{A}}+\left(\frac{166}{9}-\frac{16}{3} \zeta_{3}\right) \mathrm{C}_{\mathrm{F}}\right] \cdot \beta_{0} \\
& \left.+\left(\frac{523}{36}-72 \zeta_{3}\right) \mathrm{C}_{\mathrm{A}}^{2}+\frac{65}{3} \mathrm{C}_{\mathrm{F}} \mathrm{C}_{\mathrm{A}}+\frac{\mathrm{C}_{\mathrm{F}}^{2}}{2}\right\} \\
& +a_{s}^{4}\left(-3 C_{F}\right) \cdot c_{4} .
\end{aligned}
$$

The last known coefficient $c_{4}$ has the explicit form [6]

$$
\begin{aligned}
c_{1} \cdot c_{4}= & \left(-3 C_{F}\right) \cdot\left\{C_{A}^{3}\left(-\frac{4276}{27} \zeta_{3}+\frac{968}{9} \zeta_{3}^{2}-\frac{25090}{27} \zeta_{5}-\frac{1540}{3} \zeta_{7}+\frac{8004277}{2916}\right)\right. \\
& +n_{f} T_{r}\left[C_{A}^{2}\left(-\frac{236}{3} \zeta_{3}-\frac{704}{9} \zeta_{3}^{2}+\frac{14840}{27} \zeta_{5}+\frac{560}{3} \zeta_{7}-\frac{1238827}{486}\right)\right. \\
& \left.+C_{A} C_{\mathrm{F}}\left(\frac{20624}{27} \zeta_{3}-\frac{4400}{27} \zeta_{5}-\frac{2240}{3} \zeta_{7}+\frac{87403}{162}\right)+C_{\mathrm{F}}^{2}\left(-\frac{3608}{9} \zeta_{3}+\frac{4640}{9} \zeta_{5}-\frac{839}{27}\right)\right] \\
& +C_{A}^{2} C_{F}\left(-\frac{25456}{27} \zeta_{3}+\frac{22000}{27} \zeta_{5}+\frac{6160}{3} \zeta_{7}-\frac{1071641}{648}\right)+C_{A} C_{\mathrm{F}}^{2}\left(\frac{7768}{9} \zeta_{3}-\frac{16720}{9} \zeta_{5}+\frac{3707}{54}\right) \\
& +\left(n_{f} T_{r}\right)^{2}\left[C_{A}\left(\frac{688}{27} \zeta_{3}+\frac{128}{9} \zeta_{3}^{2}-\frac{320}{9} \zeta_{5}+\frac{165283}{243}\right)+C_{\mathrm{F}}\left(\frac{1060}{27}-\frac{928}{9} \zeta_{3}\right)\right]+C_{F}^{3}\left(32 \zeta_{3}+\frac{4823}{24}\right) \\
& \left.-n_{f} \frac{16 d_{F}^{a b c d} d_{F}^{a b c d}}{3 C_{\mathrm{F}} d_{R}}\left(13+16 \zeta_{3}-40 \zeta_{5}\right)+\frac{16 d_{F}^{a b c d} d_{A}^{a b c d}}{3 C_{\mathrm{F}} d_{R}}\left(3-4 \zeta_{3}-20 \zeta_{5}\right)-\frac{38720}{729}\left(n_{f} T_{r}\right)^{3}\right\},
\end{aligned}
$$


with the $S U_{c}(N)$-group fundamental fermion invariants

$$
\begin{aligned}
T_{r} & =\frac{1}{2} ; \quad C_{\mathrm{F}}=\frac{N^{2}-1}{2 N} ; \quad C_{A}=N ; \quad N_{A}=2 C_{\mathrm{F}} C_{\mathrm{A}} \equiv N^{2}-1 ; \\
d^{a b c} d^{a b c} & =\frac{\left(N^{2}-4\right) N_{A}}{N} ; \quad d_{F}^{a b c d} d_{A}^{a b c d}=\frac{N\left(N^{2}+6\right)}{48} N_{A} ; \\
d_{F}^{a b c d} d_{F}^{a b c d} & =\frac{N^{4}-6 N^{2}+18}{96 N^{2}} N_{A} ; \quad d_{A}^{a b c d} d_{A}^{a b c d}=\frac{N^{2}\left(N^{2}+36\right)}{24} N_{A},
\end{aligned}
$$

where $d_{R}$ is the dimension of the quark color representation, $d_{R}=3$ in QCD, and $n_{f}$ denotes the number of active flavors. The explicit form of the $\beta$-expansion for $c_{4}$ is not known yet. The numerical form of $C_{\mathrm{Bjp}}\left(a_{s}\right)$ [6] reads

$$
\begin{aligned}
C_{\mathrm{Bjp}}\left(a_{s}\right)= & 1-4\left[a_{s}+a_{s}^{2}\left(\frac{55}{3}-\frac{4}{3} n_{f}\right)+a_{s}^{3}\left(663.04-121.72 n_{f}+2.84 n_{f}^{2}\right)\right. \\
& \left.+a_{s}^{4}\left(30684.6-7897.05 n_{f}+482.64 n_{f}^{2}-6.64 n_{f}^{3}\right)\right] .
\end{aligned}
$$

\section{APPENDIX B: RG solutions for QCD charge}

1. Asymptotic freedom is the basic feature of QCD as the theory of strong interactions [34,35]. This leading order prediction was quickly complemented by the corresponding 2-loop [36,37] and 3-loop [38,39] results. The 4-loop result was obtained 17 years later [40] and here we stay on this level of accuracy. The explicit expressions for the first coefficients of $\beta$ function expansion are

$$
\begin{aligned}
\beta_{0}= & \frac{11}{3} C_{\mathrm{A}}-\frac{4}{3} T_{r} n_{f} ; \beta_{1}=\frac{34}{3} C_{\mathrm{A}}^{2}-\left(4 C_{\mathrm{F}}+\frac{20}{3} C_{\mathrm{A}}\right) T_{r} n_{f} ; \\
\beta_{2}= & \frac{2857}{54} C_{A}^{3}+2 C_{F}^{2} T_{r} n_{f}-\frac{205}{9} C_{F} C_{A} T_{r} n_{f}-\frac{1415}{27} C_{A}^{2} T_{r} n_{f}+\frac{44}{9} C_{F}\left(T_{r} n_{f}\right)^{2}+\frac{158}{27} C_{A}\left(T_{r} n_{f}\right)^{2} \\
\beta_{3}= & C_{A}^{4}\left(\frac{150653}{486}-\frac{44}{9} \zeta_{3}\right)+C_{A}^{3} T_{R} n_{f}\left(-\frac{39143}{81}+\frac{136}{3} \zeta_{3}\right)+C_{F}^{2} T_{R}^{2} n_{f}^{2}\left(\frac{1352}{27}-\frac{704}{9} \zeta_{3}\right) \\
& +C_{A} C_{F} T_{R}^{2} n_{f}^{2}\left(\frac{17152}{243}+\frac{448}{9} \zeta_{3}\right)+C_{A} C_{F}^{2} T_{R} n_{f}\left(-\frac{4204}{27}+\frac{352}{9} \zeta_{3}\right)+\frac{424}{243} C_{A} T_{R}^{3} n_{f}^{3} \\
& +C_{A}^{2} C_{F} T_{R} n_{f}\left(\frac{7073}{243}-\frac{656}{9} \zeta_{3}\right)+C_{A}^{2} T_{R}^{2} n_{f}^{2}\left(\frac{7930}{81}+\frac{224}{9} \zeta_{3}\right)+\frac{1232}{243} C_{F} T_{R}^{3} n_{f}^{3} \\
& +46 C_{F}^{3} T_{R} n_{f}+n_{f} \frac{d_{F}^{a b c d} d_{A}^{a b c d}}{N_{A}}\left(\frac{512}{9}-\frac{1664}{3} \zeta_{3}\right)+n_{f}^{2} \frac{d_{F}^{a b c d} d_{F}^{a b c d}}{N_{A}}\left(-\frac{704}{9}+\frac{512}{3} \zeta_{3}\right)+\frac{d_{A}^{a b c d} d_{A}^{a b c d}}{N_{A}}\left(-\frac{80}{9}+\frac{704}{3} \zeta_{3}\right)
\end{aligned}
$$

The corresponding 3- and 4-loop RG equations for the coupling $A=\beta_{0} \alpha_{s} /(4 \pi)$ read

$$
\frac{d A_{(3)}}{d t}=-A_{(3)}^{2}\left[1+b_{1} A_{(3)}+b_{2} A_{(3)}^{2}\right]
$$

and

$$
\frac{d A_{(4)}}{d t}=-A_{(4)}^{2}\left[1+b_{1} A_{(4)}+b_{2} A_{(4)}^{2}+b_{3} A_{(4)}^{3}\right] \quad \text { with } \quad b_{i} \equiv \frac{\beta_{i}}{\beta_{0}^{i+1}}
$$

2. The solution of this RG equation at the 2-loop level $\left(b_{2}=b_{3}=0\right)$ assumes the form

$$
\frac{1}{A_{(2)}}+b_{1} \ln \left[\frac{A_{(2)}}{1+b_{1} A_{(2)}}\right]=t .
$$


The exact solution of Eq. (B4) can be expressed in terms of the Lambert function $W(z)$ [41] (see also [42]), defined by

$$
z=W(z) e^{W(z)}
$$

This solution has the form

$$
A_{(2)}(t)=-\frac{1}{b_{1}} \frac{1}{1+W_{-1}(z(t))},
$$

where $\quad z(t)=\left(1 / b_{1}\right) \exp \left(-1+i \pi-t / b_{1}\right)$ and the branches of the multivalued function $W$ are denoted by
$W_{k}, \quad k=0, \pm 1, \ldots$ The second-iteration solution of Eq. (B4), which provides sufficient accuracy, is

$$
\frac{1}{A_{(2)}(t)} \rightarrow \frac{1}{A_{(2)}^{\mathrm{it}-2}(t)}=t+b_{1} \ln \left[t+b_{1}+b_{1} \ln \left(t+b_{1}\right)\right] .
$$

3. The approximate solution of the renormalizationgroup equation in the 4-loop of QCD [33], where the $\beta$-function is given by Eq. (B3), assumes the asymptotic expansion

$$
\begin{aligned}
A_{(4)}(t) \simeq & \frac{1}{t}\left[1-\frac{b_{1} l}{t}+\frac{1}{t^{2}}\left(b_{1}^{2}\left(l^{2}-l-1\right)+b_{2}\right) .\right. \\
& +\frac{1}{2 t^{3}}\left(b_{1}^{3}\left(-2 l^{3}+5 l^{2}+4 l-1\right)-6 b_{1} b_{2} l+b_{3}\right)+\frac{1}{6 t^{4}}\left(b_{1}^{2} b_{2}\left(2 l^{2}-l-1\right)\right. \\
& \left.\left.+b_{1}^{4}\left(6 l^{4}-26 l^{3}-9 l^{2}+24 l+7\right)-b_{1} b_{3}(12 l+1)+10 b_{2}^{2}\right)\right]
\end{aligned}
$$

where $l=\ln (t)$.

[1] J. D. Bjorken, Phys. Rev. 148, 1467 (1966).

[2] J. D. Bjorken, Phys. Rev. D 1, 1376 (1970).

[3] J. Kodaira, S. Matsuda, T. Muta, K. Sasaki, and T. Uematsu, Phys. Rev. D 20, 627 (1979).

[4] S. G. Gorishnii and S. A. Larin, Phys. Lett. B 172, 109 (1986).

[5] S. A. Larin and J. A. M. Vermaseren, Phys. Lett. B 259, 345 (1991).

[6] P. A. Baikov, K. G. Chetyrkin, and J. H. Kuhn, Phys. Rev. Lett. 104, 132004 (2010).

[7] S. J. Brodsky, G. P. Lepage, and P. B. Mackenzie, Phys. Rev. D 28, 228 (1983).

[8] S. V. Mikhailov, J. High Energy Phys. 06 (2007) 009.

[9] A. L. Kataev and S. V. Mikhailov, Phys. Rev. D 91, 014007 (2015).

[10] A. Deur, J.-M. Shen, X.-G. Wu, S. J. Brodsky, and G. F. de Teramond, Phys. Lett. B 773, 98 (2017).

[11] D. Kotlorz, S. V. Mikhailov, O. V. Teryaev, and A. Kotlorz, Phys. Rev. D 96, 016015 (2017).

[12] D. Kotlorz, S. V. Mikhailov, O. V. Teryaev, and A. Kotlorz, AIP Conf. Proc. 2075, 080007 (2019).

[13] P. A. Baikov, K. G. Chetyrkin, and J. H. Kuhn, Phys. Rev. Lett. 101, 012002 (2008).

[14] G. Cvetič and A. L. Kataev, Phys. Rev. D 94, 014006 (2016).

[15] H.-H. Ma, X.-G. Wu, Y. Ma, S. J. Brodsky, and M. Mojaza, Phys. Rev. D 91, 094028 (2015).

[16] A. Deur, V. Burkert, J.-P. Chen, and W. Korsch, Phys. Lett. B 650, 244 (2007).
[17] A. L. Kataev and S. V. Mikhailov, J. High Energy Phys. 11 (2016) 079.

[18] M. G. Alekseev et al. (COMPASS Collaboration), Phys. Lett. B 690, 466 (2010).

[19] C. Adolph et al. (COMPASS Collaboration), Phys. Lett. B 753, 18 (2016).

[20] C. Adolph et al. (COMPASS Collaboration), Phys. Lett. B 769, 34 (2017).

[21] D. Strozik-Kotlorz, S. V. Mikhailov, O. V. Teryaev, and A. Kotlorz, J. Phys. Conf. Ser. 938, 012062 (2017).

[22] P. L. Anthony et al. (E155 Collaboration), Phys. Lett. B 493, 19 (2000).

[23] A. Deur, Y. Prok, V. Burkert, D. Crabb, F. X. Girod, K. A. Griffioen, N. Guler, S. E. Kuhn, and N. Kvaltine, Phys. Rev. D 90, 012009 (2014).

[24] S. Forte and L. Magnea, Phys. Lett. B 448, 295 (1999).

[25] D. Kotlorz and A. Kotlorz, Phys. Lett. B 644, 284 (2007).

[26] D. Kotlorz and S. V. Mikhailov, J. High Energy Phys. 06 (2014) 065.

[27] I. I. Balitsky, V. M. Braun, and A. V. Kolesnichenko, Phys. Lett. B 242, 245 (1990); 318, 648(E) (1993).

[28] A. Deur et al., Phys. Rev. D 78, 032001 (2008).

[29] R. S. Pasechnik, D. V. Shirkov, and O. V. Teryaev, Phys. Rev. D 78, 071902 (2008).

[30] R. S. Pasechnik, D. V. Shirkov, O. V. Teryaev, O. P. Solovtsova, and V.L. Khandramai, Phys. Rev. D 81, 016010 (2010).

[31] R. S. Pasechnik, J. Soffer, and O. V. Teryaev, Phys. Rev. D 82, 076007 (2010). 
[32] V. L. Khandramai, R. S. Pasechnik, D. V. Shirkov, O. P. Solovtsova, and O. V. Teryaev, Phys. Lett. B 706, 340 (2012).

[33] M. Tanabashi et al. (Particle Data Group), Phys. Rev. D 98, 030001 (2018).

[34] D. J. Gross and F. Wilczek, Phys. Rev. Lett. 30, 1343 (1973).

[35] H. D. Politzer, Phys. Rev. Lett. 30, 1346 (1973).

[36] W. E. Caswell, Phys. Rev. Lett. 33, 244 (1974).

[37] D. R. T. Jones, Nucl. Phys. B75, 531 (1974).
[38] O. V. Tarasov, A. A. Vladimirov, and A. Yu. Zharkov, Phys. Lett. 93B, 429 (1980).

[39] S. A. Larin and J. A. M. Vermaseren, Phys. Lett. B 303, 334 (1993).

[40] T. van Ritbergen, J. A. M. Vermaseren, and S. A. Larin, Phys. Lett. B 400, 379 (1997).

[41] R. M. Corless, G. H. Gonnet, D. E. G. Hare, D. J. Jeffrey, and D. E. Knuth, Adv. Comput. Math. 5, 329 (1996).

[42] B. A. Magradze, Int. J. Mod. Phys. A 15, 2715 (2000). 